\title{
Current evidence for therapeutic interventions and prognostic factors in polymyalgia rheumatica: a systematic literature review informing the 2015 European League Against Rheumatism/American College of Rheumatology recommendations for the management of polymyalgia rheumatica
}

\author{
Christian Dejaco, ${ }^{1,2}$ Yogesh P Singh, ${ }^{2}$ Pablo Perel, ${ }^{3}$ Andrew Hutchings, ${ }_{1}^{4}$ \\ Dario Camellino, ${ }^{5}$ Sarah Mackie, ${ }^{6}$ Eric L Matteson, ${ }^{7}$ Bhaskar Dasgupta ${ }^{2}$
}

\begin{abstract}
Handling editor Tore K Kvien
- Additional material is published online only. To view please visit the journal online (http://dx.doi.org/10.1136/ annrheumdis-2015-207578)
\end{abstract}

For numbered affiliations see end of article.

\section{Correspondence to} Professor Bhaskar Dasgupta, Southend University Hospital, Department of Rheumatology, Prittlewell Chase, Westcliff-onsea, Essex SSO ORY, UK;

Bhaskar.dasgupta@southend. nhs.uk

ELM and BD are co-senior authors.

Received 10 March 2015 Revised 21 May 2015 Accepted 13 July 2015

CrossMark

\section{SLinked}

- http://dx.doi.org/10.1136/ annrheumdis-2015-207492 To cite: Dejaco C, Singh YP,
Perel P, et al. Ann Rheum Dis 2015;74:1808-1817.

\section{ABSTRACT}

To summarise evidence on therapeutic interventions and prognostic factors in polymyalgia rheumatica (PMR). A systematic literature review was conducted using Ovid Medline, Embase, PubMed, CINAHL, Web of Science and the Cochrane Library (1970 through April 2014). Quality of evidence (QoE) of identified studies was appraised by Grading of Recommendations Assessment, Development and Evaluation (GRADE) (interventions) and the Quality In Prognosis Studies (QUIPS) methodologies (prognostic factors). Out of 10931 titles identified, 52 articles were finally selected. A single study indicated that an initial prednisone dose of $20 \mathrm{mg} /$ day is associated with a lower short-term relapse rate than $10 \mathrm{mg} /$ day but at the cost of a higher rate of adverse events. Another study suggested a comparable efficacy of intramuscular methylprednisolone and oral glucocorticoids (GCs) with lower cumulative GC doses and less weight gain in the former group. Moderate to high QoE (1-2 studies) indicated a benefit of methotrexate in remission rates and cumulative GC doses in early PMR. Anti-tumour necrosis factor $\alpha$ agents are ineffective for PMR treatment. Among prognostic factors, female sex, high erythrocyte sedimentation rate (ESR) and peripheral arthritis were associated in some studies with a higher relapse risk. Women and patients with high ESR also appeared to have a longer duration of treatment. Several studies of varying quality, however, failed to prove these associations. In PMR, evidence for initial GC doses and subsequent tapering regimens is limited. Intramuscular methylprednisolone and methotrexate may be effective GC sparing agents. Female sex, high ESR and peripheral arthritis at disease outset are potential risk factors for a worse prognosis.

\section{INTRODUCTION}

There is still a wide heterogeneity in the methods used to treat polymyalgia rheumatica (PMR) that may be subject to personal experience, the setting in which it is managed (ie, primary or specialty care) and to the existence and implementation of national guidelines. ${ }^{1-4}$ Treatment tailored to the individual is desirable but is hampered by the absence of reliable predictors of long-term disease outcomes.
The objective of this work was to summarise evidence on therapeutic interventions and prognostic factors in PMR informing the panel developing new European League Against Rheumatism (EULAR)/American College of Rheumatology (ACR) recommendations for the management of $\mathrm{PMR}^{4 \mathrm{a}} 4 \mathrm{~b}$

\section{METHODS}

See online supplementary file S1 for full details.

In brief, we used Grading of Recommendations Assessment, Development and Evaluation (GRADE) methodology as a framework. ${ }^{5}$ The key questions were framed in the PICO (Population, Intervention, Comparator, Outcome) format (box 1). Outcomes were retrieved from a survey among rheumatologists, general practitioners and patients (see online supplementary box S1). A sensitive systematic literature search was conducted by two investigators (CD, YPS) using Ovid MEDLINE, Embase, PubMed, CINAHL, Web of Science and the Cochrane Library databases (from January 1970 until April 2014), applying the thesaurus of PMR, text words, abbreviations and truncated text words (see online supplementary box S2 for key words used for Ovid Medline). The grey literature and clinical trial registries were reviewed and tracked to determine whether additional peer-reviewed articles not identified by the primary search had been published. We excluded all articles that did not report original data, did not study patients with PMR, or that considered patients with PMR and giant cell arteritis (GCA) as a single group. We also excluded all studies on prognostic factors investigating tests that were not routinely available and/or with a $<6$ months' follow-up. Quality appraisal of interventional and prognostic studies was performed using GRADE ${ }^{6} 7$ and the Quality in Prognostic Studies (QUIPS) tool, ${ }^{8}$ respectively. We attempted to perform meta-analyses (fixed effect methods) for interventional studies whenever possible, whereas for prognostic studies, meta-analysis was impossible because of the large heterogeneity in study design, PMR case definition, measurements and definitions of prognostic factors and outcomes as well as study quality. 
Box 1 PICO (=Population, Intervention, Comparator,

Outcome) questions

PICO questions on interventions

1. In polymyalgia rheumatica (PMR) (P), what is the effect of non-steroidal anti-inflammatory drugs and/or analgesics (I) on outcome (0) compared with glucocorticoids (C)?

2. In PMR (P), what is the effect of short duration of glucocorticoid therapy (I) on outcome (0) compared with long duration of glucocorticoid therapy $(\mathrm{C})$ ?

3. In PMR (P), what is the effect of low-dose oral glucocorticoids ( $\leq 7.5 \mathrm{mg} /$ day of prednisone equivalent) (I) on outcome (0) compared with medium dose of glucocorticoids (> $7.5 \mathrm{mg} /$ day but $\leq 30 \mathrm{mg} /$ day of prednisone equivalent) (C)?

4. In PMR (P), what is the effect of medium-dose oral glucocorticoids ( $>7.5 \mathrm{mg} /$ day but $\leq 30 \mathrm{mg} /$ day of prednisone equivalent) (I) on outcome (0) compared with high dose of glucocorticoids ( $>30 \mathrm{mg} /$ day but $\leq 100 \mathrm{mg} /$ day of prednisone equivalent) (C)?

5. In PMR (P), what is the effect of an oral glucocorticoid dose of $\geq 10 \mathrm{mg} /$ day but $\leq 20 \mathrm{mg} /$ day prednisone equivalent (I) on outcome (0) compared with a dose of $>20 \mathrm{mg}$ but $\leq 30 \mathrm{mg} /$ day of prednisone equivalent $(\mathrm{C})$ ?

6. In PMR (P), what is the effect of rapid taper of glucocorticoids (I) on outcome (0) compared with slow taper of glucocorticoids (C)?

7. In PMR (P), what is the effect of intramuscular injection of glucocorticoids (I) on outcome (0) compared with oral glucocorticoids $(\mathrm{C})$ ?

8. In PMR (P), what is the effect of administration of oral glucocorticoid therapy in divided doses (morning plus evening) (I) on outcome (0) compared with a single dose (morning only) (C)?

9. In PMR (P), what is the effect of glucocorticoids plus non-biological disease modifying antirheumatic drugs (I) on outcome (0) compared with glucocorticoids alone $(C)$ ?

10. In PMR (P), what is the effect of glucocorticoids plus biological agents (I) on outcome (0) compared with glucocorticoids alone $(C)$ ?

11. In PMR (P), what is the effect of biological agents (I) on outcome (O) compared with glucocorticoids alone (C).

12. In PMR (P), what is the effect of glucocorticoids plus non-pharmacological interventions (I) on outcome (O) compared with glucocorticoids alone (C)?

PICO questions on prognostic factors

13. In PMR (P), what is the effect of older age at diagnosis (I) on outcome $(0)$ compared with younger age $(C)$ ?

14. In PMR (P), what is the effect of female sex (I) on outcome ( 0$)$ compared with male sex $(C)$ ?

15. In PMR (P), what is the effect of high levels of inflammatory markers (ie, erythrocyte sedimentation rate and/or C-reactive protein) at diagnosis (I) on outcome (0) compared with low levels of inflammatory markers $(\mathrm{C})$ ?

16. In PMR (P), what is the effect of more active/severe disease at diagnosis (I) on outcome (0) compared with lower disease activity/severity $(C)$ ?

17. In PMR (P), what is the effect of the presence of peripheral arthritis at diagnosis (I) on outcome (0) compared with the absence of peripheral arthritis (C)?
18. In PMR (P), what is the effect of longer symptom duration at diagnosis (I) on outcome (0) compared with shorter symptom duration (C)?

19. In PMR (P), what is the effect of concomitant conditions (including cardiovascular disease, cerebrovascular disease, peripheral vascular disease, osteoporosis,

hyperlipidaemia, diabetes, hypertension, infection, cataract, glaucoma, peptic ulcer, skin disorders, adiposity, mood disturbances, cognitive disorder) at diagnosis that might be exaggerated by PMR and/or glucocorticoid therapy (I) on outcome (0) compared with the absence of these conditions $(\mathrm{C})$ ?

20. In PMR (P), what is the effect of rapid response to glucocorticoids (I) on outcome (0) compared with delayed response?

21. In PMR (P), what is the effect of shared patients' management by primary and secondary care (I) on outcome (0) compared with management in primary care only?

22. In PMR (P), what is the effect of optimal control management of patients (I) on outcome (O) compared with conventional management $(\mathrm{C})$ ?

\section{RESULTS}

Our initial and update searches yielded n=10 078 and 853 articles, respectively (figure 1). The characteristics of the 52 included studies are given in table 1 (interventions) and table 2 (prognostic factors).

No study was found describing the effect of short versus long duration of glucocorticoid (GC) therapy (PICO 2), the effect of administration of GCs in divided doses (PICO 8), the prognostic value of shared patients' management by primary and secondary care (PICO 21) and the relevance of optimal control management of patients compared with conventional management (PICO 22).

The full GRADE profile on interventions and evidence tables on prognostic factors are shown in online supplementary tables S1 and S2, respectively.

\section{Evidence for therapeutic interventions (PICOs 1-12)}

Non-steroidal anti-inflammatory drugs compared with GCs (PICO 1)

Two retrospective studies $(n=364)$ reported a lower rate of vertebral fractures $(\mathrm{RR}=0.05$ (CI 0 to 0.78$)$ ) among non-steroidal anti-inflammatory drug (NSAID) users than among GCs users, whereas no difference was found in hip and radius fractures. In addition, there was a trend towards a higher rate of cardiovascular events $(\mathrm{HR}=1.64 \quad(0.99$ to 2.70$))$ and hypertension $\left(\mathrm{RR}=1.72(0.94\right.$ to 3.13$)$ in the NSAID group. ${ }^{17} 20$ The quality of evidence (QoE) for all reported outcomes was very low.

High versus low initial GC doses (PICOs 3-5)

A single randomised controlled trial (RCT) with 39 patients with PMR investigated starting doses of $20 \mathrm{mg}$ and $10 \mathrm{mg}$ oral prednisone. ${ }^{22}$ We also identified seven retrospective studies with comparisons between $>7.5 \mathrm{mg} /$ day and $\leq 7.5 \mathrm{mg} / \mathrm{day},{ }^{9} 2026$ $>15 \mathrm{mg} /$ day and $\leq 15 \mathrm{mg} /$ day $^{12}{ }^{24-26}$ and $>30 \mathrm{mg} /$ day and $\leq 30 \mathrm{mg} /$ day oral prednisone. ${ }^{18} 26$

The RCT demonstrated a lower relapse rate at 2 months in the higher-dose group than in the lower-dose group $(\mathrm{RR}=0.16(0.04$ to 0.62 ), moderate QoE), ${ }^{22}$ whereas the retrospective studies showed contradictory results (all with very low QoE). Patients in 
Figure 1 Study flow diagram detailing the literature search. PICO, Population, Intervention, Comparator, Outcome; PMR, polymyalgia rheumatica.
10065 titles from MEDLINE, Embase, PubMed, CINAHL, Web of Science (01/1970-06/2013)

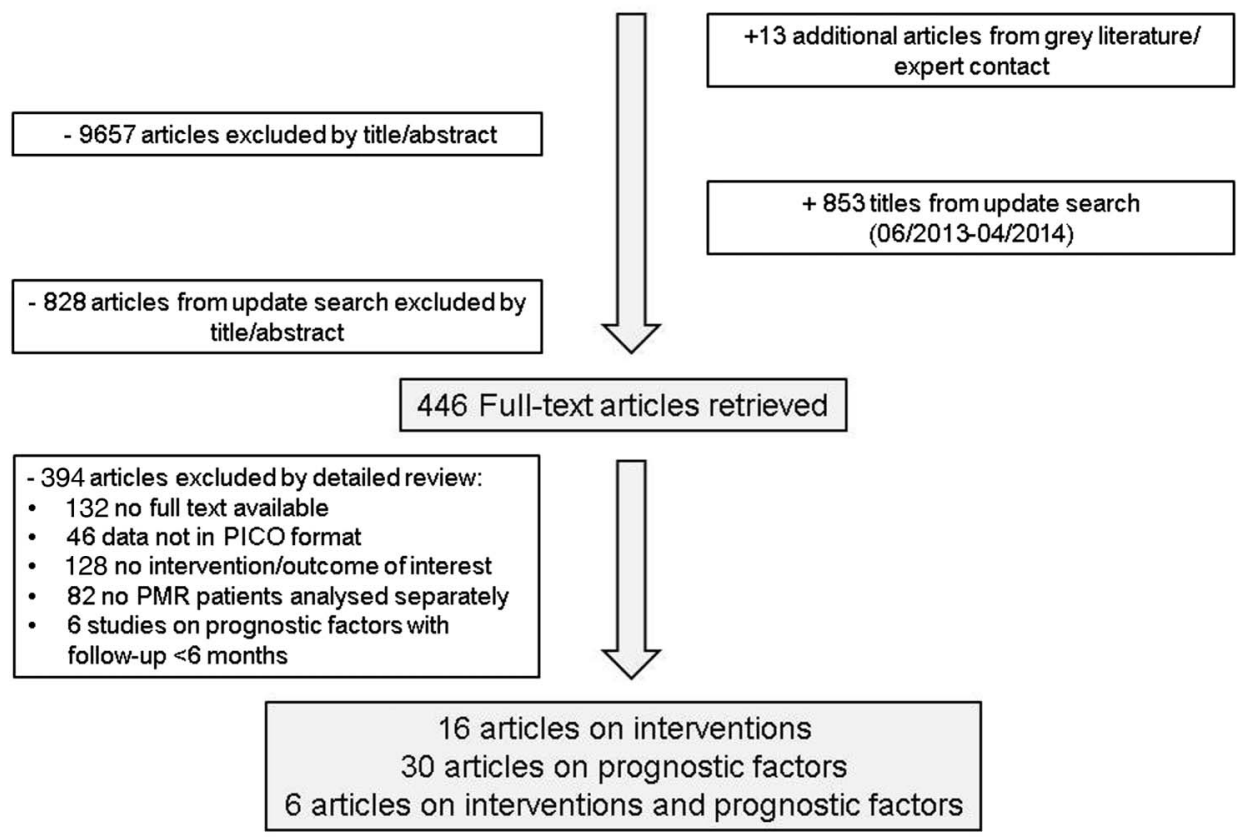

the high-dose groups, however, appeared to be at a higher risk for GC-related adverse events according to one prospective $(R R=11.55$ (0.68 to 195.63$)$, very low QoE) and one retrospective study $(\mathrm{RR}=6.73$ (1.84 to 24.56$)$, very low QoE). ${ }^{12} 22$

Rapid versus slow tapering of glucocorticoids (PICO 6)

A single retrospective study (low QoE, $n=364$ ) analysed the effect of fast versus slow GC tapering on relapse risk using statistically modelled 'tapering constants'. Compared with slow tapering, medium and fast GC dose reduction were linked with a 2.4and 5.3-fold increased risk, respectively for a first relapse. ${ }^{20}$

Intramuscular versus oral glucocorticoid therapy (PICO 7)

A single multicentre RCT (12 weeks double-blinded plus 84 weeks open-label) on 60 patients with PMR was identified. ${ }^{13}$ The authors reported a similar efficacy of intramuscular (IM) and oral GCs for remission rates at weeks 12, 48 and 96; however, the QoE decreased from moderate at first to very low at the last visit because of a lack of blinding and increasing imprecision after week 12 .

The trial reported a lower cumulative GC dose (mean difference (MD) of $1.1 \mathrm{~g}$ and $1.5 \mathrm{~g}$ at weeks 24 and 96, respectively; moderate QoE) and less weight at week 96 (MD -2.6 kg) in the IM group compared with the oral GC group. Other GC-related side effects, however, did not differ between the groups.

\section{Conventional synthetic disease-modifying antirheumatic drugs} (PICO 9)

We identified four RCTs and one retrospective study testing the use of methotrexate (MTX) at doses of $7.5-10 \mathrm{mg} /$ week. ${ }^{10} 1115242729$ In addition, a retrospective analysis on the use of hydroxychloroquine was found. ${ }^{24}$

We found moderate to high QoE from one to two studies indicating a benefit of MTX for remission at week $44(\mathrm{RR}=5.22$ $(1.28$ to 21.29$)),{ }^{27}$ relapse rate at week $76(\mathrm{RR}=0.64(0.42$ to $0.98)),{ }^{10}$ discontinuation of GCs at weeks $48(\mathrm{RR}=1.74(1.15$ to 2.64$))$ and $76(\mathrm{RR}=1.64(1.15 \text { to } 2.35))^{10}$ as well as cumulative GC doses at 12 months (meta-analysed effect of two studies: MD $0.51 \mathrm{~g}$ (0.6-0.43 g) prednisone). ${ }^{15} 27$ Additionally, Caporali et $a l^{10}$ reported median cumulative prednisone doses of $2.1 \mathrm{~g}(\mathrm{IQR}=1.96-2.9)$ and $2.97 \mathrm{~g}(2.17-3.65)$ in MTX and control groups, respectively, at week 76 .

All analyses from one to four prospective and retrospective studies reporting no benefit of MTX for these outcomes were of very low quality: ${ }^{15} 242729$ Van der Veen's study, for example, ${ }^{29}$ reported no difference in remission and relapse rates between MTX and control groups; however, this trial was limited by a very high drop-out rate (ie, study bias) of $48 \%$ (withdrawals for several reasons) and imprecision about the outcomes of interest. The (meta-analysed effect of the) two randomised studies by Ferraccioli et $a l^{15}$ and Nazarinia et $a l^{27}$ reporting no difference between groups in the relapse rate at 12 months were limited by lack of blinding, heterogeneity, indirectness (because different GC doses and relapse criteria were used) and imprecision. Additionally, the intervention and control groups in Ferraccioli's trial received different prednisone starting doses $(25 \mathrm{mg}$ in the MTX group and $15 \mathrm{mg}$ in the control arm). ${ }^{15}$

For the reduction of GC side effects, one trial reported a better dual energy X-ray absorptiometry result during follow-up in the MTX group than in the control group (MD of bone mineral density $2.7 \%$ (CI $3.9 \%$ to $1.5 \%$ ), moderate QoE) ${ }^{15}$ however, the rate of osteoporotic fractures was similar in both groups according to four studies. ${ }^{10} 111529$ None of the other GC-related adverse events tested was reduced by MTX treatment. ${ }^{10} 111529$ The QoE of these results was very low mainly because of study limitations and (serious) imprecision. None of the studies was adequately powered to detect differences in GC-related adverse events.

Hydroxychloroquine was ineffective for reducing relapses as indicated by a single very low QoE retrospective study. ${ }^{24}$

Biological agents (PICO 10 and 11)

A single 52-week RCT examined the efficacy of infliximab ( $3 \mathrm{mg} / \mathrm{kg}$ body weight) versus placebo in 53 patients with PMR. ${ }^{28}$ The trial failed to show a benefit of infliximab for primary and secondary efficacy endpoints. 


\begin{tabular}{|c|c|c|c|c|c|c|c|c|c|c|c|}
\hline Study ID & PICO & Design & Duration & Follow-up & $\begin{array}{l}\text { PMR } \\
\text { criteria }\end{array}$ & Intervention & Control & $\begin{array}{l}\text { No } \\
\text { pt. }\end{array}$ & $\begin{array}{l}\text { No female } \\
\text { patients (\%) }\end{array}$ & $\begin{array}{l}\text { No patients } \\
\text { with complete } \\
\text { follow-up (\%) }\end{array}$ & Outcomes (time point) \\
\hline 1991 Catoggio $^{9}$ & 3 & Case-control (retro.) & $7 y$ & NR & Descript. & OP $10-15 \mathrm{mg}$ & OP $7.5 \mathrm{mg}$ & 36 & $18(50)$ & $36(100)$ & Relapse (>3 m) \\
\hline $\begin{array}{l}2004 \text { Caporali }^{10} \\
2008 \text { Cimmino }^{11}\end{array}$ & 9 & R, DB, Mul.+Obs. (prosp.) & & $\begin{array}{l}76 \mathrm{w} \\
59 \mathrm{mt}\end{array}$ & Chuang & MTX $10 \mathrm{mg}+0 P 25 \mathrm{mg}$ & OP $25 \mathrm{mg}$ & 72 & $48(67)$ & $62(86)$ & $\begin{array}{l}\text { Relapse (244876 w) } \\
\text { Disc GC (244876 w) } \\
\text { GC-sides (76 w) }\end{array}$ \\
\hline $\begin{array}{l}1988 \\
\text { Delecoeuillerie }^{12}\end{array}$ & 5 & Case-control (retro.) & $10 y$ & NR & Descript. & OP $15-30 \mathrm{mg}$ & OP $7-12 \mathrm{mg}$ & 132 & NR & $132(100)$ & $\begin{array}{l}\text { Relapse (varT) } \\
\text { Disc. GC (varT) } \\
\text { Devel. GCA (varT) } \\
\text { GC-sides (varT) }\end{array}$ \\
\hline $\begin{array}{l}1998 \text { Dasgupta }^{13} \\
1997 \text { Dolan }^{14}\end{array}$ & 7 & $\begin{array}{l}\text { R, DB, Mul.+ Obs. } \\
\text { (prosp.) }\end{array}$ & & $\begin{array}{l}12 w \\
84 w\end{array}$ & Jones & $\begin{array}{l}\text { IMP } 120 \mathrm{mg} / 3 \mathrm{w} \text { (starting } \\
\text { dose) }\end{array}$ & $\begin{array}{l}\text { OP } 15 \mathrm{mg} \text { (starting } \\
\text { dose) }\end{array}$ & $60^{f}$ & $43(72)$ & $49(82)$ & $\begin{array}{l}\text { Remission (124896 w) } \\
\text { Disc GC (96 w) } \\
\text { GC-sides }(61224 \mathrm{~m}) \\
\text { Cumul GC }(24,52 \text {, } \\
96 \text { w) } \\
\text { Mortality (96 w) }\end{array}$ \\
\hline 1996 Ferraccioli $^{15}$ & 9 & R, Mul. & & $12 \mathrm{~m}$ & Descript. & MTX $10 \mathrm{mg}+$ OP $25 \mathrm{mg}$ & OP $15 \mathrm{mg}$ & 24 & $22(92)$ & $24(100)$ & $\begin{array}{l}\text { Relapse }(12 \mathrm{~m}) \\
\text { Disc GC }(12 \mathrm{~m}) \\
\text { GC-sides }(12 \mathrm{~m}) \\
\text { Cumul GC }(6,12 \mathrm{~m}) \\
\text { ESR/CRP }(12 \mathrm{~m}) \\
\text { GC-dose }<5 \mathrm{mg}(12 \mathrm{~m})\end{array}$ \\
\hline $2007 \mathrm{Fu}^{16}$ & 12 & $\mathrm{R}$ & & $12 \mathrm{w}$ & Descript. & Yanghe+OP $20 \mathrm{mg}$ & OP $20 \mathrm{mg}$ & 61 & $43(71)$ & $61(100)$ & $\begin{array}{l}\text { Remission (12 w) } \\
\text { GC-sides (12 w) } \\
\text { ESR (4812 w) } \\
\text { Morning stiff (12 w) }\end{array}$ \\
\hline 1997 Gabriel $^{17 ~ \& ~}$ & 1 & Case-control (retro.) & $22 y$ & $8 y \neq$ & Descript. & NSAIDs & $\mathrm{OP}$ & 181 & $163(89)$ & $181(100)$ & GC-sides (varT) \\
\hline 1986 Kanemaru $^{18}$ & 4 & Case-control (retro.) & $9 y$ & NR & Hamrin & OP $60 \mathrm{mg}$ & $\mathrm{OP}>10 \mathrm{mg}$ and $\leq 30 \mathrm{mg}$ & 6 & $3(50)$ & $6(100)$ & Relapse (after taper) \\
\hline 2010 Kreiner $^{19}$ & 11 & $R, D B$ & & $14 \mathrm{~d}$ & Chuang & ETN $25 \mathrm{mg} 2 \times /$ week & Placebo & 22 & NR & $20(91)$ & PMR-AS (2 w) \\
\hline \multirow[t]{2}{*}{2005 Kremers $^{20} \&$} & 3 & Case-control (retro.) & $30 y$ & $5 y \neq$ & Descript. & $\mathrm{OP}>10 \mathrm{mg}$ & $\mathrm{OP}<5 \mathrm{mg}$ & 121 & NR & $12(10)$ & Hazard 1st relapse \\
\hline & 6 & & & & & GC fast tapering & GC slow tapering & 163 & & $42(26)$ & \\
\hline 2007 Kremers $^{21}$ * & 1 & Case-control (retro.) & $30 y$ & $8 y \neq$ & Descript. & NSAIDs & $\mathrm{OP}$ & 364 & $244(67)$ & NR & GC-sides (varT) \\
\hline $1989 \mathrm{Kyle}^{22,23}$ & 5 & R+Obs. (prosp.) & NR & $\begin{array}{l}2 \mathrm{~m} \\
12-177 \mathrm{w}\end{array}$ & Jones & OP $20 \mathrm{mg}$ & OP $10 \mathrm{mg}$ & 39 & NR & $39(100)$ & $\begin{array}{l}\text { Relapse (2 m) } \\
\text { GC-sides (varT) }\end{array}$ \\
\hline \multirow[t]{2}{*}{2013 Lee $^{24}$} & 5 & Case-control (retro.) & NR & $114 \mathrm{wt}$ & Bird & $\mathrm{OP}>15 \mathrm{mg}$ & $\mathrm{OP} \leq 15 \mathrm{mg}$ & 39 & NR & $39(100)$ & Relapse (varT) \\
\hline & 9 & & & & & $\begin{array}{l}\mathrm{OP}+\mathrm{HCQ} \\
\mathrm{OP}+\mathrm{MTX}\end{array}$ & $\begin{array}{l}\mathrm{OP} \\
\mathrm{OP}\end{array}$ & $\begin{array}{c}24 \\
12\end{array}$ & & $\begin{array}{l}24(100) \\
12(100)\end{array}$ & \\
\hline 2010 Mackie $^{25}$ & 5 & Case-control (retro.) & NR & $5 y$ & Bird & $\mathrm{OP}>15 \mathrm{mg}$ & $\mathrm{OP} \leq 15 \mathrm{mg}$ & 171 & $124(73)$ & 164 (96) & $\begin{array}{l}\text { Relapse (5 y) } \\
\text { Dur. GC therapy } \\
\text { Devel. GCA (5 y) }\end{array}$ \\
\hline \multirow[t]{3}{*}{2001 Myklebust $^{26}$} & 3 & Case-control (retro.) & $8 y$ & NR & Bird/ & $\mathrm{OP}>10 \mathrm{mg}$ and $\leq 30 \mathrm{mg}$ & $\mathrm{OP} \leq 10 \mathrm{mg}$ & 175 & $124(71)$ & $157(90)$ & Disc GC (1, 2 y) \\
\hline & 4 & & & & Hamrin & $\mathrm{OP}>30 \mathrm{mg}$ & $\mathrm{OP}>10 \mathrm{mg}$ and $\leq 30 \mathrm{mg}$ & 206 & $139(68)$ & $188(91)$ & \\
\hline & 5 & & & & & $\mathrm{OP}>15 \mathrm{mg}$ and $\leq 30 \mathrm{mg}$ & $\mathrm{OP} \leq 15 \mathrm{mg}$ & 175 & $120(69)$ & $157(90)$ & \\
\hline
\end{tabular}


Another RCT compared etanercept with placebo in patients with newly diagnosed PMR not receiving GCs. ${ }^{19}$ This trial failed to show a greater reduction of the PMR activity score at week 2 with etanercept than with placebo (primary endpoint) (low QoE).

\section{Non-pharmacological interventions (PICO 12) and herbal preparations}

No clinical trials on non-pharmacological interventions were found.

For herbal preparations, we identified two randomised studies testing Chinese Yanghe herb decoction and Chinese Biqi capsules. ${ }^{16} 30$ Treatment with Chinese Yanghe resulted in a lower degree of morning stiffness and a lower erythrocyte sedimentation rate (ESR) at week 12 (low and moderate QoE, respectively). ${ }^{16}$ For Biqi capsules there was low QoE, indicating a higher response rate at week 12 using a new (not validated) clinical composite score ( $\mathrm{RR}=1.4(1.0$ to 2.0$)){ }^{30}$

\section{Evidence for prognostic factors (PICOs 13-22) \\ Older versus younger age (PICO 13)}

Four studies $(n=480$, low risk of bias (LoB) in three to six out of eight QUIPS + two categories) examined the prognostic relevance of age. ${ }^{20} 394752$ In one study, older age was associated with lower healthcare resource use $(n=364, \mathrm{LoB} 6 / 8) .{ }^{47}$

\section{Female versus male sex (PICO 14)}

The prognostic impact of sex was investigated by 21 studies $(\mathrm{n}=1811)$ with varying quality (LoB in 3-6/ 8). ${ }^{14} 182024253132 \quad 34 \quad 36-39414244 \quad 4751-535758$ A higher number of relapses in women was found in one study $(\mathrm{n}=80$, LoB $5 / 8),{ }^{37}$ whereas eight studies $(\mathrm{n}=693$, LoB ranging from 3 to 6/8) showed no such association. ${ }^{18} 202434 \quad 41515257$ One study each reported a lower discontinuation rate of GCs after 2 years $(\mathrm{n}=50, \mathrm{LoB} 4 / 8),{ }^{14}$ a longer duration of GC therapy $(\mathrm{n}=183, \mathrm{LoB} 3 / 8),{ }^{32}$ a higher risk of developing GCA $(\mathrm{n}=176$, LoB $5 / 8)^{25}$ and a higher cumulative GC dose $(\mathrm{n}=80, \operatorname{LoB} 4 /$ 8). ${ }^{37}$ Additionally, GC side effects were generally more common among women than men (three studies, total $n=196$, LoB $2-4 / 8) .313637$

Higher versus lower acute phase reactants (PICO 15)

Acute phase reactants were examined by 16 studies $(n=2067){ }^{4} 202426323339404344474850545657$ The parameter most commonly investigated was ESR, whereas C-reactive protein (six studies, $\mathrm{n}=722$ ), ${ }^{4} 2432333957$ interleukin 6 (one study, $\mathrm{n}=94)^{57}$ and plasma viscosity (one study, $\mathrm{n}=183$ ) $^{32}$ were less frequently tested. For this report, we focused on ESR as results for other acute phase reactants were comparable.

A higher ESR was associated with a higher relapse rate in three studies $\left(n=208\right.$, LoB 4-5/8), ${ }^{24} 3356$ a lower probability of GC discontinuation after 1 and 2 years in one study $(n=199$, LoB $5 / 8),{ }^{26}$ a longer duration of GC therapy in two studies $(\mathrm{n}=381, \mathrm{LoB} 2-5 / 8)^{32}{ }^{40}$ and a lower physical and mental quality of life after 1 year (one study, $n=129, \operatorname{LoB} 8 / 8$ ). ${ }^{4}$ A lack of association between baseline ESR levels and relapse rate (3 studies, $\mathrm{n}=427$, LoB 4-6/8), ${ }^{48} 5457$ discontinuation of GCs (1 study, $\mathrm{n}=20, \operatorname{LoB} 5 / 8)^{44}$ and duration of GC therapy (2 studies, $\mathrm{n}=278$, LoB $5-6 / 8)^{33} 48$ was also reported.

More active versus less active/severe disease (PICO 16)

A single high-quality study $(\mathrm{n}=129)$ examined the prognostic impact of morning stiffness on patients' reported outcomes, 


\begin{tabular}{|c|c|c|c|c|c|c|c|c|c|c|}
\hline Study ID & PICO & Design & Dur. & Follow-up & PMR criteria & $\begin{array}{l}\text { Prognostic } \\
\text { factor }\end{array}$ & No pt. & $\begin{array}{l}\text { No female } \\
(\%)\end{array}$ & $\begin{array}{l}\text { No pt. with } \\
\text { complete } \\
\text { follow-up (\%) }\end{array}$ & Outcomes (time point) \\
\hline 1985 Ayoub $^{31}$ & 14 & Case-control (retro.) & $8 y$ & NR & Descript. & Sex & 76 & $42(55)$ & $76(100)$ & GC-sides (varT) \\
\hline \multirow[t]{2}{*}{2008 Barraclough $^{32}$} & 14 & Case-control (retro.) & $10 y$ & $2 y$ & Clinical & Sex & 183 & $138(75)$ & $183(100)$ & Longer duration GC therapy \\
\hline & 15 & & & & & ESR, CRP, PV & 180 & $138(77)$ & $180(100)$ & \\
\hline 2000 Cantini $^{33} ¥$ & 15 & Obs. (prosp.) & $5 y$ & $37 \mathrm{~m} \neq$ & Descript. & ESR & 177 & $117(66)$ & $177(100)$ & Duration GC therapy \\
\hline 1996 Caplanne $^{34}$ & 14 & Obs. (prosp.) & NR & NR & Bird & Sex & 20 & $15(75)$ & $20(100)$ & Relapse (varT) \\
\hline 2006 Ceccato $^{35}$ & 17 & Case-control (retro.) & $13 y$ & $40 \mathrm{~m} \ddagger$ & Chuang & Arthritis & 74 & $56(76)$ & $74(100)$ & Relapse (varT) \\
\hline 1994 Cimmino $^{36 ~ \# ~}$ & 14 & Obs. (prosp.) & NR & $19 \mathrm{~m}^{\dagger}$ & Jones \& Hazleman & Sex & 40 & $24(60)$ & $38(95)$ & GC-sides (varT) \\
\hline 2006 Cimmino $^{37} \#$ & 14 & Obs. (prosp.) & NR & $15 \mathrm{~m}^{\dagger}$ & Chuang & Sex & 80 & $52(65)$ & $80(100)$ & $\begin{array}{l}\text { Number of relapses (varT), Cumul GC (varT), } \\
\text { GC-sides (varT) }\end{array}$ \\
\hline \multirow[t]{3}{*}{2011 Cimmino $^{38 ~ \# ~}$} & 14 & Obs. (prosp.) & $18 \mathrm{~m}$ & $6 \mathrm{~m}$ & Bird & Sex & 60 & $35(58)$ & $60(100)$ & Response (1 m) \\
\hline & 17 & & & & & Arthritis & & & & Response (1 m) \\
\hline & 20 & & & & & Rapid response & & & & Relapse (6 m) \\
\hline 1997 Dolan $^{14}$ & 14 & Obs. (prosp.) & NR & $96 \mathrm{w}$ & Jones & Sex & 50 & $36(72)$ & $50(100)$ & Disc GC (2 y) \\
\hline \multirow[t]{4}{*}{2013 Do-Nguyen ${ }^{39}$} & 13 & Case-control (retro.) & $11 \mathrm{y}$ & $1 \mathrm{y} \ddagger$ & NR & Age & 100 & $71(71)$ & $100(100)$ & Remission on and off therapy (1 y) \\
\hline & 14 & & & & & Sex & & & & \\
\hline & 15 & & & & & ESR & & & & \\
\hline & 18 & & & & & sympt. dur. & & & & \\
\hline 1997 Gonzalez-Gay ${ }^{40}$ & 15 & Case-control (retro.) & NR & $27 / 32 \mathrm{mt}$ & Descript. & ESR & 201 & $121(60)$ & $191(95)$ & Duration GC therapy \\
\hline 1999 Gonzalez-Gayy & 14 & Case-control (retro.) & NR & $\geq 12 \mathrm{~m}$ & Descrip. & Sex & 134 & $85(63)$ & NR & Relapse (varT) \\
\hline $2001 \mathrm{Gran}^{42}$ & 14 & Case-control (retro.) & $11 y$ & $64 \mathrm{~m}^{\dagger}$ & Bird & Sex & 274 & $183(67)$ & NR & Mortality (varT) \\
\hline 1996 Helfgott $^{43}$ & 15 & Case-control (retro.) & $5 y$ & NR & Jones & ESR & 117 & $89(76)$ & $117(100)$ & Time to response \\
\hline \multirow[t]{3}{*}{2007 Hutchings $^{4}$} & 15 & Obs. (prosp.) & $2 y$ & $12 \mathrm{~m}$ & Jones & ESR & 129 & $77(60)$ & $122(95)$ & HAQ (12 m), SF36-PCS/MCS (12 m) \\
\hline & 16 & & & & & Morning stiffness & & & & HAQ (12 m), SF36-PCS/MCS (12 m) \\
\hline & 20 & & & & & Rapid response & & & & Relapse (12 m) \\
\hline \multirow[t]{2}{*}{1986 Kanemaru $^{18}$} & 14 & Case-control (retro.) & $9 y$ & NR & Hamrin & Sex & 6 & $3(50)$ & $6(100)$ & Relapse (varT) \\
\hline & 20 & & & & & Rapid response & & & & \\
\hline \multirow[t]{2}{*}{1997 Kanik $^{44}$} & 14 & Case-control (retro.) & NR & $33-38 \mathrm{mt}$ & Descript. & Sex & 20 & $16(80)$ & $20(100)$ & Disc GC (varT) \\
\hline & 15 & & & & & ESR & & & & \\
\hline \multirow[t]{2}{*}{$2012 \mathrm{Kim}^{45}$} & 17 & Case-control (retro.) & NR & $28 \mathrm{mt}$ & Bird & Arthritis & 51 & $36(71)$ & $41(80)$ & Remission (varT) \\
\hline & 19 & & & & & comorbidities & & & & \\
\hline 2012 Kimura $^{46}$ & 17 & Case-control (retro.) & $10 y$ & $26 \mathrm{mt}$ & Hunder & RS3PE & 151 & $78(52)$ & $136(90)$ & Relapse (varT), GC-sides (varT), duration GC therapy \\
\hline \multirow[t]{3}{*}{2005 Kremers $^{20} \&$} & 13 & Case-control (retro.) & $30 y$ & $5 y \ddagger$ & Descript. & Age & 364 & $244(67)$ & $163^{*}(100)$ & Hazard 1st relapse \\
\hline & 14 & & & & & Sex & & & & \\
\hline & 15 & & & & & ESR & & & & \\
\hline \multirow[t]{3}{*}{2005 Kremers $^{47}$ \& } & 13 & Case-control (retro.) & $30 y$ & $5 y \neq$ & Descript. & Age & 364 & $244(67)$ & $364(100)$ & HealthC (6 m, ever) \\
\hline & 14 & & & & & Sex & & & & HealthC (1 m, $6 \mathrm{~m}$, ever) \\
\hline & 15 & & & & & ESR & & & & HealthC (6 m, ever) \\
\hline
\end{tabular}


Table 2 Continued

\begin{tabular}{|c|c|c|c|c|c|c|c|c|c|c|}
\hline Study ID & PICO & Design & Dur. & Follow-up & PMR criteria & $\begin{array}{l}\text { Prognostic } \\
\text { factor }\end{array}$ & No pt. & $\begin{array}{l}\text { No female } \\
(\%)\end{array}$ & $\begin{array}{l}\text { No pt. with } \\
\text { complete } \\
\text { follow-up (\%) }\end{array}$ & Outcomes (time point) \\
\hline 2000 Larrosa $^{48}$ & 15 & Case-control (retro.) & $8 y$ & NR & Chuang & ESR & 101 & $67(66)$ & $97(96)$ & Relapse (varT) \\
\hline \multirow[t]{3}{*}{2013 Lee $^{24}$} & 14 & Case-control (retro.) & NR & $114 w^{\dagger}$ & Bird & Sex & 39 & $28(72)$ & $39(100)$ & Relapse (varT) \\
\hline & 15 & & & & & ESR & & & & \\
\hline & 17 & & & & & Arthritis & & & & \\
\hline 2010 Mackie $^{25}$ & 14 & Obs. (prosp.) & NR & $5 y$ & Bird & Sex & 176 & $124(71)$ & $164(93)$ & Earlier disc GC therapy, Devel. GCA (varT) \\
\hline \multirow[t]{3}{*}{2012 Mazzantini ${ }^{49}$} & 19 & Case-control (retro.) & $39 y$ & NR & Bird & Osteoporosis & 222 & $154(69)$ & NR & Fractures (varT) \\
\hline & & & & & & Diabetes & & & & Cardiovascular events (varT) \\
\hline & & & & & & Dyslipidaemia & & & & Cardiovascular events (varT) \\
\hline \multirow[t]{2}{*}{2000 Meyerhof $^{50}$} & 14 & Case-control (retro.) & NR & $563 d^{\dagger}$ & Bird & ESR & 22 & $15(68)$ & $14(64)$ & Response to GC (varT) \\
\hline & 20 & & & & & Fast taper & & & & Remission (varT), Relapse (varT) \\
\hline 2001 Myklebust $^{26}$ & 15 & Case-control (retro.) & $8 y$ & NR & Bird & ESR & 199 & $160(80)$ & $180(91)$ & Disc GC $(1,2 y)$ \\
\hline 2000 Nagaoka $^{51}$ & 14 & Case-control (retro.) & $12 y$ & $5 y \ddagger$ & Bird & Sex & 20 & $10(50)$ & $18(90)$ & Relapse (varT) \\
\hline \multirow[t]{2}{*}{1971 Paulsen $^{52}$} & 13 & Case-control (retro.) & NR & $41 \mathrm{mt}$ & NR & Age & 16 & $14(88)$ & $16(100)$ & Relapse (varT) \\
\hline & 14 & & & & & Sex & & & & \\
\hline 1999 Prickard $^{53}$ & 14 & Case-control (retro.) & $13 y$ & NR & Descript. & Sex & 49 & $40(82)$ & $37(76)$ & $\begin{array}{l}\text { Remission (2 y), Osteoporosis (varT), diabetes (varT), } \\
\text { weight gain (varT), cataract (varT), moon face (varT), } \\
\text { gastric complications (varT) }\end{array}$ \\
\hline 1999 Proven $^{54} \&$ & 15 & Case-control (retro.) & 21 & $5-7 y \ddagger$ & Descript. & ESR & 232 & $163(70)$ & $232(100)$ & Remission (varT), relapse (varT) \\
\hline 1998 Salvarani $^{55} ¥$ & 17 & Obs. (prosp.) & $5 y$ & $25-41 \mathrm{~m}^{\dagger}$ & Healey & $\begin{array}{l}\text { Arthritis } \\
\text { RS3PE }\end{array}$ & 177 & $117(66)$ & $177(100)$ & Relapse (varT), duration GC therapy, Cumul GC (varT) \\
\hline 1999 Salvarani $^{56} ¥$ & 15 & Obs. (prosp.) & NR & $44 \mathrm{mt}$ & Descript. & ESR & 92 & $69(75)$ & $91(99)$ & Relapse (varT) \\
\hline \multirow[t]{3}{*}{2005 Salvarani $^{57} ¥$} & 14 & Obs. (prosp.) & $4 y$ & $35 \mathrm{m \neq}$ & Descript. & Sex & 94 & $70(75)$ & $94(100)$ & Relapse (varT) \\
\hline & 15 & & & & & ESR & & & & \\
\hline & 17 & & & & & Arthritis & & & & \\
\hline 1995 Schaufelberger $^{58}$ & 14 & Case-control (retro.) & $4 y$ & $36 \mathrm{mt}$ & Descript. & Sex & 222 & $158(71)$ & $222(100)$ & Mortality (varT) \\
\hline 1995 Schreiber $^{59}$ & 20 & Obs. (prosp.) & NR & $38 \mathrm{~m}^{\ddagger}$ & Descript. & $\begin{array}{l}\text { Normal CRP within } \\
1 \text { week }\end{array}$ & 20 & $11(55)$ & $12-7^{5}(60-35)$ & Disc GC $(2,3,4,5$ y), GC-sides (varT) \\
\hline
\end{tabular}

Articles containing both, data on interventions and prognostic factors are marked in bold letters.

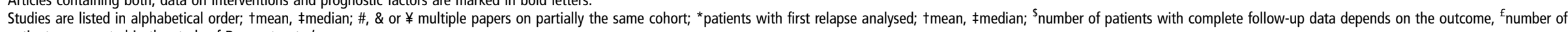
patients as reported in the study of Dasgupta et al.

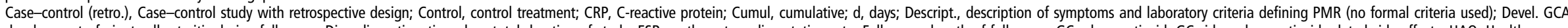
development of giant cell arteritis during follow-up; Disc, discontinuation; dur., total duration of study; ESR, erythrocyte sedimentation rate; Follow-up, length of follow-up; GC, glucocorticoid; GC-sides, glucocorticoid-related side effects; HAQ, Health Assessment Questionnaire, HealthC, healthcare resource use, inc., increased; m, months; MCS, mental component summary score; Mul., multicenter; No pt., number of patients; No, number of; NR; not reported; NSAIDs; non-steroidal anti-inflammatory

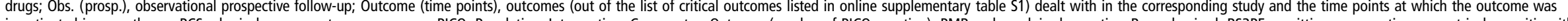

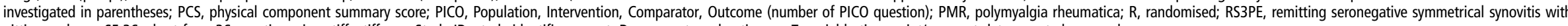
pitting oedema; SF-36, short form 36 questionnaire; stiff, stiffness; Study ID, study identifier; sympt. Dur., symptom duration; varT, variable time point/no exact date reported; w, weeks; y, years. 
demonstrating that a longer duration of morning stiffness at baseline was linked with worse function and quality of life at 12 months. $^{4}$

Presence versus absence of peripheral arthritis (PICO 17)

The prognostic value of peripheral arthritis was investigated by seven studies $\left(n=645\right.$, LoB 2-6/8). ${ }^{24} 353845465557$ The presence of peripheral arthritis was associated with a higher risk of relapse according to one study $(\mathrm{n}=177$, LoB $5 / 8),{ }^{55}$ whereas three studies $(n=207$, LoB 5-6/8) did not confirm this observation. $^{24} 3555$

Longer versus shorter symptom duration (PICO 18)

A single study $(n=100$, LoB $3 / 8)$ examined the prognostic impact of symptom duration before PMR diagnosis, reporting no association of this factor with later remission. ${ }^{39}$

Presence versus absence of conditions exaggerated by PMR and/ or glucocorticoid therapy (PICO 19)

Two studies $(n=273$, LoB 2-4/8) investigated the prognostic impact of a total set of four concomitant conditions (depression, osteoporosis, diabetes and dyslipidaemia), reporting no significant results. $^{45} 49$

Rapid versus delayed response to glucocorticoids (PICO 20)

A rapid response to GC therapy was dealt with by five studies $\left(n=237\right.$, LoB 2-6/8). ${ }^{4} 18385059$ A rapid decline of C-reactive protein was associated with a lower risk of GC side effects in a single low-quality study, ${ }^{59}$ whereas treatment response was irrelevant for remission and relapse rates and for duration of GC therapy. 418385059

\section{DISCUSSION}

One of our most intriguing observations is that fundamental treatment principles of PMR such as initial GC doses, tapering schedules and duration of treatment have not been examined by high-quality trials thus far. In contrast, we found moderate to good evidence that MTX is of benefit for patients with a new diagnosis of PMR. ${ }^{10} 152729$ Interestingly, it is clinical practice to prescribe MTX to patients with GC-resistant disease, although this approach is not supported by published evidence. ${ }^{2}{ }^{3}$ The clinical value of other conventional disease-modifying antirheumatic drugs for treatment of PMR is still unclear.

The MTX dose used in PMR trials was lower than the dose normally used in other rheumatic diseases (particularly rheumatoid arthritis); ${ }^{60}$ and it is difficult to compare the relative efficacy of MTX between PMR and other conditions, because outcome parameters are disease specific, and disease course and concomitant treatments vary across diseases. ${ }^{61}$ Whether higher MTX doses are more effective but still safe for treatment of PMR has to be clarified by future studies. ${ }^{62}$

For biological agents, evidence suggests that tumour necrosis factor $\alpha$ blocking agents are not effective in PMR, thus contrasting the promising results of earlier small case series. ${ }^{63-66}$ Tocilizumab, used in a few cases thus far, ${ }^{67}{ }^{68}$ is currently being studied in a phase 2 trial (clinicaltrials.gov NCT01396317) and results of a study comparing secukinumab, canakinumab and GCs (clinicaltrials.gov NCT01364389) will be available soon.

The evidence supporting the value of prognostic factors is only fair to moderate because of methodological limitations of most studies and because the associations were not consistently reported by all authors. A single study, for example, showed a higher relapse rate in women than in men, ${ }^{37}$ whereas eight other studies found no such association. ${ }^{18} 20243441515257 \mathrm{On}$ the other hand, women appeared to have a longer duration of treatment, $^{14}{ }^{32}$ higher cumulative GC doses ${ }^{37}$ and more GC-related side effects. ${ }^{31} 3637$ Whether female patients benefit from a closer (than standard) clinical monitoring, a lower initial GC dose and a lower threshold for using MTX has to be clarified by future clinical studies.

We found one earlier systematic literature review on interventions in PMR reporting similar conclusions to our report for initial GC doses, IM methylprednisolone and the value of MTX. ${ }^{69}$ That review, however, was limited by a less comprehensive literature search than carried out in our study (eg, Embase was not searched, non-English articles were excluded), by a lack of a priori formulated key questions and outcomes, as well as by the inclusion of studies analysing patients with GCA and PMR as a single group. ${ }^{69}$ Additionally, the authors used the Jadad scale for quality appraisal, although this method has explicitly been discouraged for systematic literature reviews. ${ }^{70} 71$

In summary, moderate- to high-quality data support the use of IM GCs and MTX as GC sparing agents in PMR, whereas several treatment aspects such as initial GC doses and tapering regimen have not, or only inadequately, been investigated. Female sex, high ESR and the presence of peripheral arthritis were associated with a worse prognosis of PMR; however, a number of studies also failed to prove these associations.

\section{Author affiliations}

${ }^{1}$ Department of Rheumatology, Medical University Graz, Graz, Austria

2Department of Rheumatology, Southend University Hospital, Southend, UK

${ }^{3}$ Epidemiology \& Population Health Faculty, London School of Hygiene \& Tropical Medicine, London, UK

${ }^{4}$ Department of Health Services Research \& Policy, London School of Hygiene \& Tropical Medicine, London, UK

${ }^{5}$ Department of Internal Medicine, Research Laboratory and Academic Division of Clinical Rheumatology, University of Genova, Genova, Italy

${ }^{6}$ NIHR-Leeds Musculoskeletal Biomedical Research Unit, University of Leeds, Leeds Institute of Rheumatic and Musculoskeletal Medicine, Leeds, UK

${ }^{7}$ Division of Rheumatology, Department of Health Sciences Research, Mayo Clinic

College of Medicine, Rochester, MN, USA

\section{Twitter Follow Sarah Mackie at @Sarah_L_Mackie}

Acknowledgements We thank Catherine Wardle from Southend library for her assistance in retrieving full-text articles.

Contributors All authors contributed to the conception and design of the study, acquisition and/or interpretation of data, drafting of the article or revising it critically for important intellectual content and approved the final version of the manuscript. All authors had full access to all of the data (including statistical reports and tables) in the study and can take responsibility for the integrity of the data and the accuracy of the data analysis.

Funding Supported by research grants from the American College of Rheumatology and the European League Against Rheumatism. The funder had a role neither in the conduction of the systematic literature review nor in the interpretation of data.

Competing interests YPS, PP, AH, DC, CD—consultation and/or speaking engagements ('R'): BMS, Pfizer, AbbVie, MSD, Merck Serono, Roche; research funding ('F'): Pfizer, MSD. SM-R: Pfizer, Napp Pharmaceuticals, AstraZeneca. ELM -F: Ardea, Sanofi, Centocor-Jansen, Celgene, Amgen, Roche, Genentech, Mesoblast, Novartis, Pfizer. BD—R: Merck, F: Health Technology Assessment UK, Roche, Mundipharma, Servier.

\section{Patient consent Obtained.}

Provenance and peer review Not commissioned; externally peer reviewed.

\section{REFERENCES}

1 Helliwell T, Hider SL, Mallen CD. Polymyalgia rheumatica: diagnosis, prescribing, and monitoring in general practice. Br J Gen Pract 2013;63:e361-6.

2 Dasgupta B, Borg FA, Hassan N, et al. BSR and BHPR guidelines for the management of polymyalgia rheumatica. Rheumatology (Oxford) 2010;49: 186-90.

3 Schmidt WA, Gromnica Ihle E. Polymyalgia rheumatica und Riesenzellarteriitis (Arteriitis temporalis). In: Busse 0, Fleig W, Mayet W, et al., eds. Rationelle 
Diagnostik und Therapie in der Inneren Medizin: Leitlinien-basierte Empfehlungen für die Praxis. Elsevier Inc, 2011; Chapter 5.2.

4 Hutchings A, Hollywood J, Lamping DL, et al. Clinical outcomes, quality of life, and diagnostic uncertainty in the first year of polymyalgia rheumatica. Arthritis Rheum 2007;57:803-9.

4a Dejaco C, Singh YP, Perel P, et al. 2015 Recommendations for Management of Polymyalgia Rheumatica: An European League Against Rheumatism and American College of Rheumatology Collaborative Initiative. Ann Rheum Dis 2015;74: 1799-1807.

4b Dejaco C, Singh YP, Perel P, et al. 2015 Recommendations for Management of Polymyalgia Rheumatica: An European League Against Rheumatism and American College of Rheumatology Collaborative Initiative. Arthritis Rheumatol 2015. (in press).

5 Guyatt G, Oxman AD, Akl EA, et al. GRADE guidelines: 1. Introduction-GRADE evidence profiles and summary of findings tables. I Clin Epidemiol 2011;64:383-94.

6 Balshem $H$, Helfand $M$, Schünemann HJ, et al. GRADE guidelines: 3. Rating the quality of evidence. J Clin Epidemiol 2011;64:401-6.

7 Group GW. GRADE homepage. http://www.gradeworkinggroup.org/index.htm

8 Hayden JA, Coté P, Bombardier C. Evaluation of the quality of prognosis studies in systematic reviews. Ann Intern Med 2006;144:427-37.

9 Catoggio W, Soriano ER, Imamura PM. Treatment of polymyalgia rheumatica: lower initial dose. Br J Rheumatol 1991;30:393-5.

10 Caporali R, Cimmino MA, Ferraccioli G, et al. Prednisone plus methotrexate for polymyalgia rheumatica: a randomized, double-blind, placebo-controlled trial. Ann Intern Med 2004;141:493-500.

11 Cimmino MA, Salvarani C, Macchioni P, et al. Long-term follow-up of polymyalgia rheumatica patients treated with methotrexate and steroids. Clin Exp Rheumatol 2008;26:395-400.

12 Delecoeuillerie G, Joly P, Cohen de Lara A, et al. Polymyalgia rheumatica and temporal arteritis: a retrospective analysis of prognostic features and different corticosteroid regimens (11 year survey of 210 patients). Ann Rheum Dis 1988;47:733-9.

13 Dasgupta B, Dolan AL, Panayi GS, et al. An initially double-blind controlled 96 week trial of depot methylprednisolone against oral prednisolone in the treatment of polymyalgia rheumatica. Br J Rheumatol 1998;37:189-95

14 Dolan AL, Moniz C, Dasgupta B, et al. Effects of inflammation and treatment on bone turnover and bone mass in polymyalgia rheumatica. Arthritis Rheum 1997:40:2022-9.

15 Ferraccioli G, Salaffi F, De Vita S, et al. Methotrexate in polymyalgia rheumatica: preliminary results of an open, randomized study. J Rheumatol 1996:23:624-8.

$16 \mathrm{Fu} \mathrm{HZ}$. [Clinical observation on effect of modified Yanghe Decoction combined with glycocorticoid for treatment of polymyalgia rheumatica]. Zhongguo Zhong Xi Yi Jie He Za Zhi 2007:27:894-7.

17 Gabriel SE, Sunku J, Salvarani C, et al. Adverse outcomes of antiinflammatory therapy among patients with polymyalgia rheumatica. Arthritis Rheum 1997;40: 1873-8.

18 Kanemaru K, Nagura H, Ooyama T, et al. [Report of 6 cases with polymyalgia rheumatica and a review of the literature]. Nihon Ronen Igakkai Zasshi 1986;23:469-76

19 Kreiner F, Galbo H. Effect of etanercept in polymyalgia rheumatica: a randomized controlled trial. Arthritis Res Ther 2010;12:R176.

20 Kremers HM, Reinalda MS, Crowson CS, et al. Relapse in a population based cohort of patients with polymyalgia rheumatica. J Rheumatol 2005;32:65-73.

21 Maradit Kremers H, Reinalda MS, Crowson CS, et al. Glucocorticoids and cardiovascular and cerebrovascular events in polymyalgia rheumatica. Arthritis Rheum 2007:57:279-86.

22 Kyle V, Hazleman BL. Treatment of polymyalgia rheumatica and giant cell arteritis. I. Steroid regimens in the first two months. Ann Rheum Dis 1989;48:658-61.

23 Kyle V, Hazleman BL. Treatment of polymyalgia rheumatica and giant cell arteritis. II. Relation between steroid dose and steroid associated side effects. Ann Rheum Dis 1989;48:662-6

24 Lee JH, Choi ST, Kim JS, et al. Clinical characteristics and prognostic factors for relapse in patients with polymyalgia rheumatica (PMR). Rheumatol Int 2013:33:1475-80.

25 Mackie SL, Hensor EM, Haugeberg G, et al. Can the prognosis of polymyalgia rheumatica be predicted at disease onset? Results from a 5 -year prospective study. Rheumatology (Oxford) 2010;49:716-22.

26 Myklebust G, Gran JT. Prednisolone maintenance dose in relation to starting dose in the treatment of polymyalgia rheumatica and temporal arteritis. A prospective two-year study in 273 patients. Scand I Rheumatol 2001;30: 260-7.

27 Nazarinia AM, Moghimi J, Toussi J. Efficacy of methotrexate in patients with polymyalgia rheumatica. Koomesh 2013;14:265-70.

28 Salvarani C, Macchioni P, Manzini C, et al. Infliximab plus prednisone or placebo plus prednisone for the initial treatment of polymyalgia rheumatica: a randomized trial. Ann Intern Med 2007;146:631-9.
29 Van der Veen MJ, Dinant HJ, van Booma-Frankfort C, et al. Can methotrexate be used as a steroid sparing agent in the treatment of polymyalgia rheumatica and giant cell arteritis? Ann Rheum Dis 1996;55:218-23.

30 Wang B, Zhang B, Wang H. Clinical observation about 18 cases of polymyalgia rheumatica by using Biqi capsule. China J Tradit Chin Med Pharm 2009;24:596-8.

31 Ayoub WT, Franklin CM, Torretti D. Polymyalgia rheumatica. Duration of therapy and long-term outcome. Am J Med 1985;79:309-15.

32 Barraclough $\mathrm{K}$, Liddell WG, du Toit J, et al. Polymyalgia rheumatica in primary care: a cohort study of the diagnostic criteria and outcome. Fam Pract 2008;25:328-33.

33 Cantini F, Salvarani C, Olivieri I, et al. Erythrocyte sedimentation rate and C-reactive protein in the evaluation of disease activity and severity in polymyalgia rheumatica: a prospective follow-up study. Semin Arthritis Rheum 2000;30:17-24.

34 Caplanne D, Le Parc JM, Alexandre JA. Interleukin-6 in clinical relapses of polymyalgia rheumatica and giant cell arteritis. Ann Rheum Dis 1996;55:403-4.

35 Ceccato F, Roverano SG, Papasidero S, et al. Peripheral musculoskeletal manifestations in polymyalgia rheumatica. JCR J Clin Rheumatol 2006;12:167-71.

36 Cimmino MA, Moggiana G, Montecucco C, et al. Long term treatment of polymyalgia rheumatica with deflazacort. Ann Rheum Dis 1994:53:331-3.

37 Cimmino MA, Parodi M, Caporali R, et al. Is the course of steroid-treated polymyalgia rheumatica more severe in women? Ann N Y Acad Sci 2006;1069:315-21.

38 Cimmino MA, Parodi M, Montecucco C, et al. The correct prednisone starting dose in polymyalgia rheumatica is related to body weight but not to disease severity. BMC Musculoskelet Disord 2011;12:94.

39 Do-Nguyen D, Inderjeeth C, Edelman J, et al. Retrospective analysis of the clinical course of patients treated for polymyalgia. Open Access Rheumatol Res Rev 2013:5:33-41.

40 Gonzalez-Gay MA, Rodriguez-Valverde V, Blanco R, et al. Polymyalgia rheumatica without significantly increased erythrocyte sedimentation rate. A more benign syndrome. Arch Intern Med 1997;157:317-20.

41 González-Gay MA, García-Porrúa C, Vázquez-Caruncho $\mathrm{M}$, et al. The spectrum of polymyalgia rheumatica in northwestern Spain: incidence and analysis of variables associated with relapse in a 10 year study. J Rheumatol 1999;26:1326-32.

42 Gran JT, Myklebust G, Wilsgaard T, et al. Survival in polymyalgia rheumatica and temporal arteritis: a study of 398 cases and matched population controls. Rheumatology (Oxford) 2001;40:1238-42.

43 Helfgott SM, Kieval RI. Polymyalgia rheumatica in patients with a normal erythrocyte sedimentation rate. Arthritis Rheum 1996;39:304-7.

44 Kanik KS, Bridgeford PH, Germain BF, et al. Polymyalgia rheumatica with a low erythrocyte sedimentation rate: comparison of 10 cases with 10 cases with high erythrocyte sedimentation rate. J Clin Rheumatol 1997;3:319-23.

$45 \mathrm{Kim} \mathrm{HA}$, Lee J, Ha YJ, et al. Induction of remission is difficult due to frequent relapse during tapering steroids in Korean patients with polymyalgia rheumatica. J Korean Med Sci 2012;27:22-6.

46 Kimura M, Tokuda Y, Oshiawa $\mathrm{H}$, et al. Clinical characteristics of patients with remitting seronegative symmetrical synovitis with pitting edema compared to patients with pure polymyalgia rheumatica. J Rheumatol 2012:39:148-53.

47 Kremers HM, Reinalda MS, Crowson CS, et al. Use of physician services in a population-based cohort of patients with polymyalgia rheumatica over the course of their disease. Arthritis Rheum 2005;53:395-403.

48 Larrosa M, Gratacos J, Sala M. Polymyalgia rheumatica with low erythrocyte sedimentation rate at diagnosis. J Rheumatol 2000;27:1815-16.

49 Mazzantini M, Torre C, Miccoli M, et al. Adverse events during longterm low-dose glucocorticoid treatment of polymyalgia rheumatica: a retrospective study. J Rheumatol 2012;39:552-7.

50 Meyerhoff J. Evaluating an alternative oral regimen for the treatment of polymyalgia rheumatica. J Clin Rheumatol 2000;6:61.

51 Nagaoka S, Ohno M, Ohno S, et al. Long-term outcome for patients with polymyalgia rheumatica. Rinshou Ryumachi 2000;12:348-52.

52 Paulsen S. [Polymyalgia rheumatica. Long term treatment with steroids]. Ugeskr Laeger 1971;133:944-5.

53 Prickarts M, Lagro-Janssen T, Lagro-Janssen ALM. Polymyalgia rheumatica in four general practices. [Dutch] Polymyalgia rheumatica in vier huisartspraktijken. Huisarts Wet 1999;42:597-601.

54 Proven A, Gabriel SE, O'Fallon WM, et al. Polymyalgia rheumatica with low erythrocyte sedimentation rate at diagnosis. J Rheumatol 1999;26:1333-7.

55 Salvarani C, Cantini F, Macchioni P, et al. Distal musculoskeletal manifestations in polymyalgia rheumatica: a prospective followup study. Arthritis Rheum 1998;41:1221-6

56 Salvarani C, Boiardi L, Mantovani V, et al. HLA-DRB1 alleles associated with polymyalgia rheumatica in northern Italy: correlation with disease severity. Ann Rheum Dis 1999;58:303-8.

57 Salvarani C, Cantini F, Niccoli L, et al. Acute-phase reactants and the risk of relapse/recurrence in polymyalgia rheumatica: a prospective followup study. Arthritis Rheum 2005;53:33-8.

58 Schaufelberger C, Bengtsson BA, Andersson R. Epidemiology and mortality in 220 patients with polymyalgia rheumatica. Br J Rheumatol 1995;34:261-4. 
59 Schreiber S, Buyse M. The CRP initial response to treatment as prognostic factor in patients with polymyalgia rheumatica. Clin Rheumatol 1995;14:315-18.

60 Smolen JS, Landewé R, Breedveld FC, et al. EULAR recommendations for the management of rheumatoid arthritis with synthetic and biological disease-modifying antirheumatic drugs: 2013 update. Ann Rheum Dis 2014;73:492-509.

61 Lopez-Olivo MA, Siddhanamatha HR, Shea B, et al. Methotrexate for treating rheumatoid arthritis. Cochrane database Syst Rev 2014;6:CD000957.

62 Díaz-Borjón A. Guidelines for the use of conventional and newer disease-modifying antirheumatic drugs in elderly patients with rheumatoid arthritis. Drugs Aging 2009;26:273-93.

63 Salvarani C, Cantini F, Niccoli L, et al. Treatment of refractory polymyalgia rheumatica with infliximab: a pilot study. J Rheumatol 2003;30:760-3.

64 Migliore A, Massafra U, Carloni E, et al. TNF-alpha blockade induce clinical remission in patients affected by polymyalgia rheumatica associated to diabetes mellitus and/or osteoporosis: a seven cases report. Eur Rev Med Pharmacol Sci 2005;9:373-8.
65 Corrao S, Pistone $G$, Scaglione R, et al. Fast recovery with etanercept in patients affected by polymyalgia rheumatica and decompensated diabetes: a case-series study. Clin Rheumatol 2009;28:89-92.

66 Catanoso MG, Macchioni P, Boiardi L, et al. Treatment of refractory polymyalgia rheumatica with etanercept: an open pilot study. Arthritis Rheum 2007;57:1514-19.

67 Al Rashidi A, Hegazi MO, Mohammad SA, et al. Effective control of polymyalgia rheumatica with tocilizumab. I Clin Rheumatol 2013;19:400-1.

68 Macchioni P, Boiardi L, Catanoso M, et al. Tocilizumab for polymyalgia rheumatica: report of two cases and review of the literature. Semin Arthritis Rheum 2013:43:113-18.

69 Hernandez-Rodriguez J, Cid MC, Lopez-Soto A, et al. Treatment of polymyalgia rheumatica: a systematic review. Arch Intern Med 2009;169:1839-50.

70 Higgins J, Green S. Cochrane Handbook for Systematic Reviews of Interventions Version 5.1.0 [updated March 2011]. The Cochrane Collaboration, 2011.

71 Jadad AR, Moore RA, Carroll D, et al. Assessing the quality of reports of randomized clinical trials: is blinding necessary? Control Clin Trials 1996;17:1-12. 